\title{
MobETX: uma métrica de roteamento ciente da mobilidade para Internet das Coisas
}

\author{
Gabriel Augusto R. dos Reis ${ }^{1}$, Bruno P. Santos ${ }^{1}$, Luiz F. M. Vieira ${ }^{2}$ \\ ${ }^{1}$ Instituto de Ciências Exatas e Aplicadas (Decsi) \\ Universidade Federal de Ouro Preto (UFOP) - Brasil \\ ${ }^{2}$ Departamento de Ciência da Computação (DCC) \\ Universidade Federal de Minas Gerais (UFMG) - Brasil \\ gabriel.requena@aluno.ufop.edu.br \\ bruno.ps@ufop.edu.br, lfvieira@dcc.ufmg.br
}

\begin{abstract}
The routing process in Internet of Things (IoT) is a key aspect when devices can move. It enables devices to communicate with each other, enhancing functionalities of everyday objects where they are attached. However, the mobility cause frequent topology changes turning the routing process a challenge. Most IoT's routing protocols rely on a Link Quality Estimator (LQE) to weigh links and choose routes. The Expected Transmission Count (ETX) commonly is a good LQE for wireless links between static devices, but when devices move ETX fails. In this paper, we present Mobile Expected Transmission Count (MobETX) as a new metric to qualify multi-hop wireless links for mobile scenarios. MobETX keeps all features from ETX, but uses the devices' mobility metrics to assist the routing process of choosing routes. In our experiments, the routing protocol by using MobETX is at least $5 \%$ better in package delivery than ETX in hard mobile scenarios and deliver $>98 \%$ of packets on static scenarios.
\end{abstract}

Resumo. O processo de roteamento na Internet of Things (IoT) é um aspecto chave quando dispositivos podem mover. $O$ roteamento habilita a comunicação entre os dispositivos, potencializando a funcionalidade dos objetos do dia-adia a eles anexados. Contudo, a mobilidade causa frequentes mudanças na topologia tornando o roteamento um desafio. A maioria dos protocolos de roteamento da IoT usam um estimador de enlace (Link Quality Estimator (LQE)) no processo de escolha de rotas, sendo o Expected Transmission Count (ETX) um dos mais utilizados para ponderar enlaces sem fio entre dispositivos estáticos. Contudo, o ETX não funciona bem quando os dispositivos se movem. Neste artigo, apresentamos o Mobile Expected Transmission Count (MobETX) como estimador para enlaces sem fio entre dispositivos móveis. MobETX mantém as características do ETX, mas usa métricas de mobilidade dos dispositivos para dar assistência no processo de escolha de rotas. Em nossos experimentos, o protocolo de roteamento usando MobETX apresentou melhora de ao menos $5 \%$ na taxa de entrega de pacotes quando comparado com ETX em cenários de alta mobilidade e $>98 \%$ de entrega de pacotes em cenários estáticos.

\section{Introdução}

Internet of Things (IoT) é um conceito que vem ganhando força no cenário moderno, sua ideia básica é potencializar as funcionalidades dos objetos do dia a dia através da 
comunicação (tipicamente sem fio), do uso de sensores de contexto e atuadores para mobilidade [Atzori et al. 2010, Kassab and Darabkh 2020, Santos et al. 2016]. Com o avanço da tecnologia, a IoT se torna ubíqua, nos possibilitando, monitorar o nosso contexto, com o diferencial de estarmos conectados em redes trocando informações, o que facilita as tomadas de decisões ou obtenção de respostas a um determinado evento.

Para viabilizar esses benefícios, destacamos a construção e manutenção eficiente das rotas entre os dispositivos, como uma das principais subtarefas de suporte a IoT. O Routing Protocol for Low-Power and Lossy Networks (RPL) ${ }^{1}$ é um protocolo de roteamento com suporte ao Internet Protocol version 6 (IPv6) frequentemente utilizado na IoT. O RPL é baseado em vetor de distâncias que utiliza um estimador de qualidade de enlace, referenciados aqui pelo termo em inglês Link Quality Estimator (LQE), que visa classificar os enlaces sem fio em uma determinada janela de tempo. Em [Baccour et al. 2012] são apresentadas duas categorias de LQEs: Os LQEs baseados em hardware ou software. Os LQEs baseados em hardware, obtêm as informações diretamente do transceptor de rádio (por exemplo, o rádio CC2420 [Instruments 2007] e similares), por outro lado, os LQEs baseados em software aplicam algum método numérico ou estatístico sobre o envio, recepção e/ou confirmação de pacotes. Naturalmente, os LQEs baseados em software são amplamente empregados devido ao seu fácil projeto, implantação e custo associado.

Atualmente, o Expected Transmission Count (ETX) [De Couto et al. 2005] tem notoriedade como LQE baseado em software devido às suas características. O ETX evita enlaces de maior perda, penaliza rotas com mais saltos e considera enlaces assimétricos. O ETX tem sido empregado em diferentes implementações de protocolos de roteamento ao longo do tempo [Santos et al. 2017, Santos et al. 2018, Kassab and Darabkh 2020, Santos et al. 2020], além disso, o ETX foi estendido de diferentes modos para acomodar situações onde o ETX original não se encaixava bem, como em situações com diferentes taxas de transmissão de dados, dispositivos com mais de um transceptor de rádio ou ainda quando a rede apresenta alguma dinâmica [Bahr et al. 2006, Draves et al. 2004].

Neste trabalho, damos destaque ao cenário móvel. A mobilidade está presente no dia a dia e isso torna a vida mais fácil e os aplicativos da IoT mais flexíveis. Quando um dispositivo se move, há várias métricas de mobilidade que podem ser extraídas, por exemplo, velocidade do nó, tempos de espera, distância de viagem, contatos potenciais, pontos de interesse, entropia de movimento e outros. Em redes, especialmente no ponto de vista de roteamento, a mobilidade implica em mudanças frequentes na topologia, que podem requerer atualizações de rotas, para refletir a nova organização topológica. Embora já existam diversas métricas de roteamento, atualmente essas métricas apenas qualificam os enlaces sem fio de acordo com o LQE que, no melhor de nossos conhecimentos, não levam em conta as características de mobilidade dos dispositivos.

Diante do acima exposto, a principal contribuição deste trabalho é a proposição de um LQE chamado de Mobile Expected Transmission Count (MobETX), o qual incorpora características de mobilidade dos dispositivos ao ETX. O objetivo do estimador MobETX é melhorar a construção de rotas dos protocolos de roteamento (ex.: RPL) mesmo sob eventos de mobilidade dos dispositivos. Este trabalho também introduz o conceito da categoria de LQEs híbridos, estendendo a classificação proposta em [Baccour et al. 2012].

${ }^{1}$ RFC 6550 
A categoria de LQEs híbridos, abrange os LQEs que são simultaneamente baseados em hardware e software, como o MobETX. Em nossos experimentos, o MobETX apresentou melhora na taxa de entrega de pacotes de ao menos $5 \%$, em situações com dispositivos móveis, quando comparada com outras métricas (ex.: Métricas baseadas em hop-count e ETX). E em cenários estáticos, o MobETX se comporta como o ETX entregando > 98\% dos pacotes quando uma rota existe e sem uso de mecanismos de confirmação.

Este trabalho está disposto de acordo com a seguinte estrutura: A seção 2 apresenta os trabalhos relacionados. Na seção 3 é apresentado o LQE híbrido MobETX. A seção 4 são apresentados os cenários dos experimentos, além dos detalhes de implementação e configuração e ao fim disserta sobre os resultados obtidos dos experimentos, por fim, a seção 5 conclui o artigo e apresenta os trabalhos futuros.

\section{Trabalhos relacionados}

Esta seção inicia com uma breve introdução sobre o protocolo de roteamento RPL, bem como sua intersecção com o estimador MobETX. Na sequência, LQEs propostos na literatura são colocados em perspectiva e o MobETX é contextualizado.

\subsection{O RPL em poucas palavras}

Em 2012, o Routing over Low-power and Lossy networks (RoLL) working group, um grupo que gerencia os tópicos de roteamento em IoT, padronizou o RPL 1 como o protocolo de roteamento para Low-power and Lossy Networkss (LLNs) [Sobral et al. 2019]. O RPL constrói uma estrutura chamada de Destination-Oriented Directed Acyclic Graphs (DODAG) para representar a rede como exibe a Figura 1(a). Um DODAG não possui ciclos e pode ser aterrado (grounded) ou flutuante (floating), isto é, aqueles que atendem aos requisitos de conectividade e os que não.

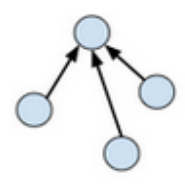

(a) Exemplos de DODAGs

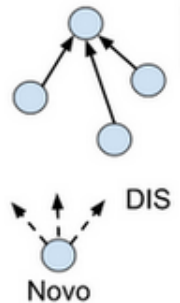

(b)

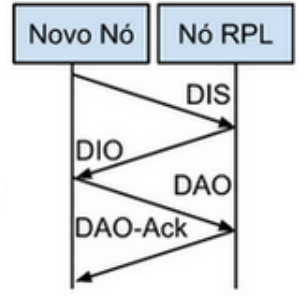

(c)

Figura 1. RPL - Entrada de um novo nó e mensagens de controle

O RPL usa quatro mensagens de controle, sendo elas: i) DODAG Information Solicitation (DIS) utilizada para solicitar entrada em uma instância DODAG (veja a Figura 1(b), ii) DODAG Information Object (DIO) para permitir que outros nós aprendam sobre DODAGs existentes e possam se juntar à estrutura; iii) Destination Advertisement Object (DAO) é uma solicitação de acesso ao DODAG que é enviada de um nó filho para seus pais ou raiz do DODAG e; iv) DAO-Ack (DAO-Ack) que nada mais é que a confirmação de aceite ou não a uma solicitação de entrada no DODAG do RPL. A Figura 1(c) apresenta o processo de entrada de um novo nó a uma instância do RPL. 
O RPL é um protocolo genérico que se adapta a uma variedade de tipos de rede ao usar diferentes Funções Objetivo (OFs) Uma OF declara o resultado do processo usado por um nó RPL para selecionar rotas em uma instância RPL com base nos DIOs recebidos ${ }^{3}$ Em resumo, uma $\mathrm{OF}$ é usada para selecionar nós que serão roteadores (isto é, nós que servem como pais ou possíveis sucessores) em direção à raiz da estrutura DODAG. Também, o RPL usa a OF para computar o rank de um dispositivo. Este rank é uma distância lógica entre a raiz e o nó. O rank é trocado entre os nós, através das mensagens de controle e essa informação é útil para evitar loops, por exemplo.

Uma OF, em consonância com as especificações do RPL, descreve como um nó selecionará parentes (na estrutura DODAG) em potencial a partir de sua vizinhança. A meta é oferecer uma boa conectividade. Uma OF deve ser projetada para encontrar a raiz mais próxima, para tanto, um pai e um conjunto de possíveis pais são selecionados caso seja possível. Então, o tráfego em direção à raiz é encaminhado via o parente escolhido.

$$
p p_{\text {novo }}=\left\{\begin{array}{cc}
p c, & s e\left(p c<p p_{\text {atual }}-\lambda\right) . \\
p p_{\text {atual }}, & \text { caso contrário }
\end{array}\right.
$$

Na RFC 6552ㅁ é apresentada a Função Objetivo Zero (OF0) como uma OF básica para encontrar o rank. Essa métrica é similar ao hop-count, a qual computa a distância em saltos entre um nó e a raiz do DODAG. Porém, em LLNs, nem sempre o menor caminho em saltos, representa a melhor rota, por isso, é recomendado que estimadores, tais como o ETX [De Couto et al. 2005], sejam usados como base do processo para a computação do rank de um nó. Neste sentido, outras funções objetivo existem como, por exemplo, a Minimum Rank Objective Function with Hysteresis (MRHOF), de forma simplificada, a MRHOF define o rank dos nós como a soma do menor rank possível configurado para a rede com o custo estimado do enlace atual, rank zero para a raiz ou o valor máximo definido para a rede em caso de nós sem rota para a raiz. Mais detalhes da MRHOF podem ser vistos na RFC6719 [Gnawali and Levis 2012]. A escolha do novo pai preferido $\left(p p_{\text {novo }}\right)$ é feita através da comparação do custo da métrica do possível pai candidato $(p c)$ com o pai preferido atual $\left(p p_{\text {atual }}\right)$, tal como descrito na Equação 1 . Observe que é indicado um limiar $(\lambda)$ na Equação 1 que serve como fronteira para a troca de pai preferido, a esse processo é dado o nome de Hysteresis. Vale notar que outro LQE pode ser utilizado para escolha de novo pai, desde que sejam respeitadas as regras do RPL e da OF. Nesta direção, este trabalho aborda uma adaptação da OF para utilizar no MobETX.

Uma alternativa ao se tratar de cenários móveis, é alterar o mecanismo de identificação dos nós pais ou alterar o próprio RPL, sem modificar o LQE usado, como pode ser visto em [Fotouhi et al. 2015, Oliveira and Vazao 2016, Santos et al. 2020].

\subsection{Classificação dos LQEs}

Diversos LQEs foram propostos ao longo do tempo como mostra a Figura 2. Em [Baccour et al. 2012], os autores propõem uma classificação para os LQEs até então existentes. A princípio, os LQEs foram classificados como aqueles baseados em hardware ou em software (observe a Figura 3). Nós também concordamos com tal classificação e

\footnotetext{
${ }^{2}$ RFC6552 [Thubert et al. 2012]

${ }^{3}$ Uma Função Objetivo não é um algoritmo.
} 
taxonomia. Porém, vamos além ao estendê-la para acomodar também LQEs recentes, em um grupo que chamamos de LQEs híbridos, isto é, aqueles que estão na interseção entre uso de hardware e software. No melhor de nossos conhecimentos esses LQEs não consideram características de mobilidade. A seguir, apresentamos esses tipos de LQEs.

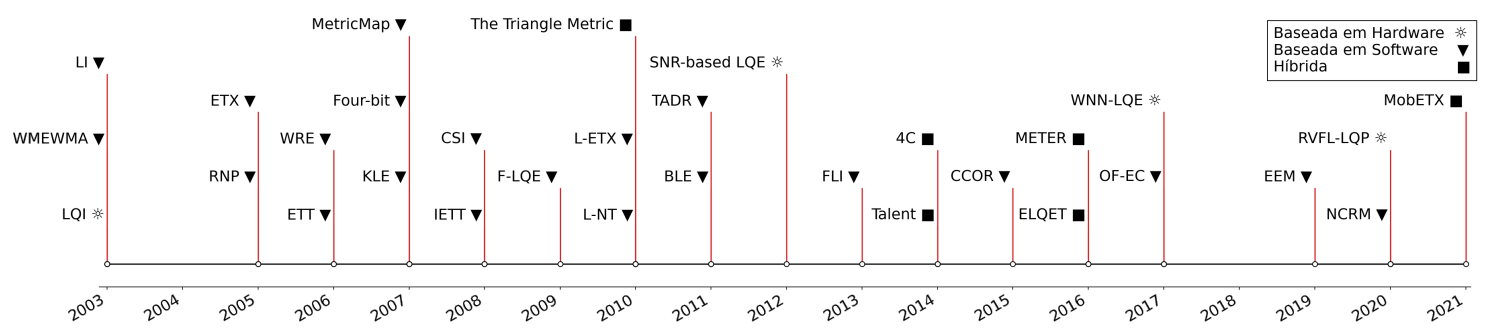

Figura 2. Linha do tempo dos LQEs.

Os LQEs baseados em hardware leem as informações do sinal recebido diretamente do transceptor de rádio para determinar a qualidade do enlace. A principal vantagem desta abordagem é a sua simplicidade e a não necessidade de computação adicional. Entretanto, esses LQEs leem apenas pacotes recebidos, tornando difícil, por exemplo, a classificação de enlaces com alta taxa de perda de pacotes e, como consequência, podem superestimar os enlaces. Detalhes dos LQEs apresentados na Figura 3 podem ser encontrados em [Baccour et al. 2012, Tan et al. 2012, Sun et al. 2017, Xue et al. 2020].

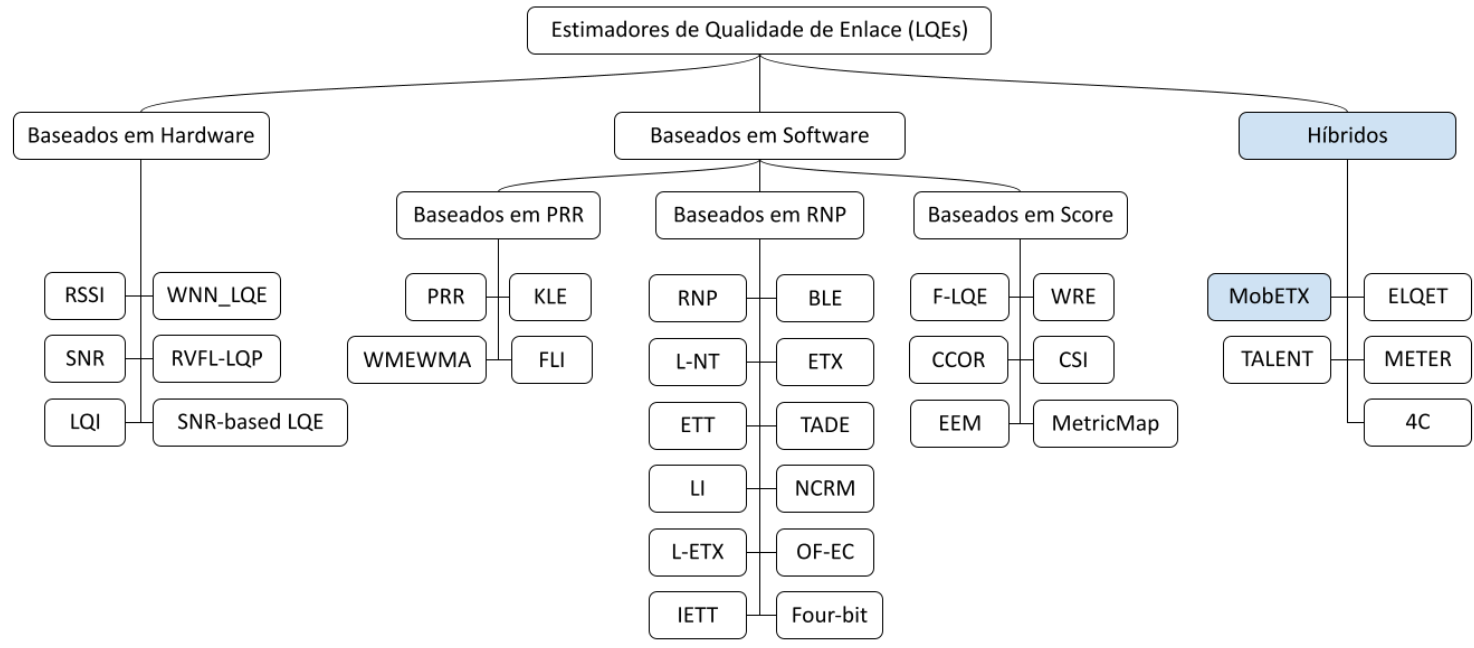

Figura 3. Classificação dos LQEs.

Por outro lado, existem os LQEs baseados em software, subdivididos em: i) LQEs baseados em Packet Reception Rate (PRR); ii) LQEs baseados em Required Number of Packet retransmissions (RNP) e; iii) LQEs baseados em score. Comentados a seguir:

i) LQEs baseados em PRR: Consideram a taxa de recepção de pacotes. São implementados do lado do receptor, simples de medir e têm sido amplamente usados em protocolos de roteamento. Sua eficiência está diretamente ligada à janela de tempo definida para realizar as medições. Nesta categoria podemos citar o fuzzy logic based link quality indicator (FLI) [Guo et al. 2013], que utiliza lógica Fuzzy, tendo 
como entrada o PRR, seu coeficiente de variância e uma métrica própria que representa a confiabilidade dos enlaces. Além desta existem outras, como demonstra a Figura 3 e outros detalhes podem ser encontrados em [Baccour et al. 2012].

ii) LQEs baseados em RNP: Consideram o número requerido de transmissões de um pacote. São implementados do lado do emissor, o qual deve contar o número médio de transmissões/retransmissões de pacotes necessários antes da recepção bem-sucedida. Particularidades destes LQEs podem ser encontradas em [Charles and Palanisamy 2020, Bahr et al. 2006, Lowrance and Lauf 2017, Lamaazi and Benamar 2017, Alizai et al. 2011, Ren et al. 2011].

iii) LQEs baseados em SCORE: Consideram informações obtidas a partir do software do dispositivo (Ex.: Taxa de recepção de pacotes, estabilidade do enlace, nível de assimetria do enlace, ruído do canal, localização, distância dos nós, e outros). Implementados do lado do emissor, utilizam os dados obtidos e critérios próprios, para fornecerem uma pontuação ou um rótulo de qualidade ao enlace. Alguns LQEs desta categoria são: Fuzzy link Quality Estimator (F-LQE), The Weighted Regression Estimator (WRE), MetricMap, Channel State Information (CSI) além de Optimizing Routing Based on Congestion Control (CCOR) e Energy Estimation Metric (EEM). Maiores detalhes podem ser encontrados em [Baccour et al. 2012, Ding et al. 2016, Pereira et al. 2019].

Neste trabalho, introduziremos o conceito de LQEs híbridos de forma a estender a classificação apresentada em [Baccour et al. 2012]. Os LQEs híbridos, são aqui propostos para acomodar LQEs que simultaneamente usam informações de hardware e software no auxílio da construção de rotas (Ex.: Na computação do rank do nó e escolha dos possíveis pais do RPL). O MobETX, se enquadra neste quesito assim como outras propostas recentes ${ }^{4}$ (vide Figura 3). A principal diferença da nossa abordagem, é que o MobETX considera fatores da mobilidade dos dispositivos, tais como velocidade do nó, duração e quantidade de enlaces ao longo da mobilidade dos nós.

\subsection{Métricas de mobilidade}

Métricas de mobilidade são medidas quantitativas que representam diversos aspectos da mobilidade humana e podem ser extraídas durante a movimentação de um dispositivo. Essas métricas são usadas para analisar o comportamento dos dispositivos ao longo de sua rota. Algumas destas métricas serão escolhidas para serem usadas no MobETX.

As métricas de mobilidade podem ser categorizadas em sociais, espaciais e temporais. As métricas sociais estão diretamente relacionadas à conectividade dos dispositivos, nos ajudam a explicar como os dispositivos se conectam entre si e algumas propriedades dessa conexão; As métricas espaciais estão relacionadas com o movimento do dispositivo e nos fornecem uma noção sobre as distâncias percorridas por cada nó; Por fim, métricas temporais, estão relacionadas à rotina dos dispositivos e nos indicam como ocorre a repetição da movimentação dos nós. Para aprofundamento sobre os conceitos apresentados ou detalhamentos de métricas nas devidas categorias, recomendamos a leitura de [University of Osnabrück 2016, Souza et al. 2018].

${ }^{4}$ Detalhes dos LQEs híbridos (Figura 3] podem ser encontrados em [Boano et al. 2010, Jayasri and Hemalatha 2017, Liu et al. 2016, Liu and Cerpa 2014b, Liu and Cerpa 2014a] 
As métricas utilizadas no MobETX são: Velocidade média (espacial); Duração média do enlace (social) e Número total de enlaces (social). Todas as métricas utilizadas se referem do início até o presente momento da rede e serão detalhadas na seção 3.2 .

\section{O estimador MobETX}

\subsection{Background}

O ETX, um LQE baseado em software, é um dos LQEs com maior notoriedade atualmente [Kassab and Darabkh 2020]. Ele foi desenvolvido como uma alternativa ao HopCount. Isso porque notou-se que em comunicações sem fio, nem sempre a menor rota (em número de saltos) provê o melhor desempenho na comunicação. Neste sentido, o objetivo do ETX é auxiliar no cálculo da rota com o menor número de transmissões e retransmissões, levando em consideração a perda de pacotes e a assimetria dos enlaces sem fio. O ETX é calculado através da Equação 2, onde $d_{f}$ é a probabilidade do pacote ser entregue com sucesso e $d_{r}$ é a probabilidade da confirmação ser recebida [De Couto et al. 2005].

$$
\frac{1}{d_{f} \times d_{r}}
$$

Os autores do ETX mostram que os protocolos de roteamento que a usam, possuem maior rendimento em redes sem fio de múltiplos saltos. Isso ocorre, pois o ETX minimiza o número esperado de transmissões e retransmissões, para entregar um pacote com sucesso. As principais características do ETX são: Considerar a taxa de entrega de pacotes bem como assimetria dos enlaces; Penalizar rotas com mais saltos; Minimizar o uso da banda com mensagens de controle desnecessárias e; Diminuir a energia gasta na rede, pois minimiza o número de transmissões por pacote.

\subsection{Um estimador híbrido: MobETX}

Apresentamos aqui o MobETX, um estimador de enlace que se difere dos anteriormente citados, pois utiliza informações da mobilidade do dispositivo (que podem ser obtidas através de sensores, por exemplo, o GPS), juntamente com o ETX, a fim de estimar a qualidade do enlace, mantendo todas as características do ETX citadas previamente, projetado para ser utilizado em cenários com padrões de movimentação similar ao humano ou em cenários com grande variação de velocidade nos dispositivos.

Para estimar a mobilidade dos dispositivos, utilizamos métricas de mobilidade que estão presentes nas Equações 3 e 4 , representando a média de tempo de duração dos enlaces realizados pelo dispositivo e a grandeza de sua velocidade média com relação a velocidade máxima (definida a priori) que pode ser alcançada na rede respectivamente, onde (i) é o dispositivo em questão. Na equação 3, $n$ é um enlace feito pelo nó $i$, a duração do enlace é obtida através da camada de enlace e $\Omega$ é o conjunto de todos os enlaces feitos pelo nó. Na Equação 4, $v_{i \text { avg }}$ indica a velocidade média do dispositivo e $v_{\max }$ é a velocidade máxima que os dispositivos podem alcançar na rede. A partir das equações obtemos a Estimativa de Mobilidade (EM) representada na Equação 5, onde $\tau$ se refere ao tempo que o dispositivo está conectado na rede e $\alpha$ é um valor entre $[0,1]$ responsável por balancear as Equações 3 e 4 . É importante observar que estas métricas de mobilidade foram escolhidas devido à sua fácil obtenção pelos nós, baixo custo e 
processamento e a não necessidade de solicitar dados aos nós vizinhos o que, com outras métricas de mobilidade, poderia acarretar em mais transmissões de controle. Dentre a métricas utilizadas, apenas a velocidade, em $v_{i \text { avg }}$, é obtida através de hardware.

$$
\begin{gathered}
\Delta_{i}=\frac{\sum_{n \in \Omega_{i}} \text { duração }_{n}}{\left|\Omega_{i}\right|} \quad(3) \quad v_{i}=\left\{\begin{array}{cc}
0, & \text { se } v_{\text {max }}=0 . \\
\frac{v_{i} \text { avg }}{v_{\max }}, & \text { caso contrário }
\end{array}\right. \\
E M_{i}=\left(\left(1-\left(\alpha \cdot\left(\frac{\Delta_{i}}{\tau}\right)\right)\right)+\left((1-\alpha) \cdot v_{i}\right)\right) \\
M o b E T X=((\beta \cdot E T X)+((1-\beta) \cdot E M \cdot \gamma))
\end{gathered}
$$

O MobETX é definido na Equação 6 e considera tanto o ETX quanto a EM. Na equação aparece o termo $\beta$, o qual é usado para ajustar o peso entre o ETX e a EM. Essas métricas possuem escalas diferentes, sendo assim $\gamma$ é usado para ajuste da EM.

Para a implementação do MobETX, sugerimos a utilização da Mobile Expected Transmission Count - Objective Function (MobETX-OF) conforme Equação 7. A MobETX-OF é similar à MRHOF, mas utilizando-se o MobETX ao invés do ETX. A MobETX-OF seleciona seu nó pai a partir da comparação entre todos os nós de uma lista de possíveis pais, comparando dois a dois, os Ranks destes possíveis pais e escolhendo o menor valor entre eles. Para que haja uma troca eficiente de nó pai, é recomendado que se utilize o limiar mínimo de 8, ou seja, a troca, somente é realizada caso o Rank deste seja 8 unidades menor que o Rank do nó pai atual. Após a escolha, o dispositivo calcula seu próprio Rank a partir da Equação 7, onde $R_{a n k} k_{b a s e}$ é o valor de rank inicial de cada nó e Rank $k_{\text {incremento }}$ é o valor adicionado a cada salto para evitar laços de roteamento. É recomendado que os valores de $\operatorname{Rank}_{\text {base }}$ e Rank $k_{\text {incremento }}$ sejam 128, como discutido na RFC6719 [Gnawali and Levis 2012] e definido como padrão do Contiki-OS. Após a definição do Rank atual do nó, este valor é divulgado a todos os nós vizinhos.

$$
\text { Rank }=\text { Rank }_{\text {base }}+\text { Rank }_{\text {incremento }}+\text { MobETX }
$$

\section{Materiais e Métodos}

Nesta seção iremos abordar os métodos utilizados para realizar os experimentos e a avaliação dos resultados obtidos. Como ambiente de experimentação foi usado emulador de redes Cooja presente no Contiki-OS 3.0 [Dunkels et al. 2004]. Os parâmetros utilizados para simulação são descritos na Tabela 1 , demais parâmetros foram mantidos como no padrão do sistema Contiki-OS.

Para estudar o efeito da mobilidade na taxa de entrega de dados usando os estimadores MobETX e ETX usamos um esquema de experimento fatorial $2^{k}$. A mobilidade dos dispositivos é de fato um fator que influencia no desempenho das métricas que estimam a qualidade dos enlaces, sendo, portanto, um fator que será considerado. Ademais, na confecção do MobETX, três outros fatores possuem destaque e podem impactar no seu desempenho, sendo eles: O limiar $\lambda$ para troca do pai preferido (vide Equação 1), o $\alpha$ da 


\begin{tabular}{|c|c|}
\hline \multicolumn{2}{|l|}{ Parâmetros do Contiki/Cooja } \\
\hline \# de nós & 100 \\
\hline Modelo de conectividade & UDGM \\
\hline Dimensão & $1000 \mathrm{~m}^{2}$ \\
\hline Tipos de nós & Cooja Mote \\
\hline Protocolos & RPL, Trickle \\
\hline Intervalo de transmissão de pacotes & $300 \mathrm{~s}$ \\
\hline Randomização de transmissão de pacotes & $300 \mathrm{~s}$ \\
\hline Tempo de simulação & $24 \mathrm{~h}$ \\
\hline
\end{tabular}

\begin{tabular}{clccc}
\hline$\#$ & Mobilidade & $\lambda$ & $\alpha$ & $\beta$ \\
\hline 1 & estático & 8 & 0.3 & 0.5 \\
2 & estático & 16 & 0.3 & 0.5 \\
3 & estático & 8 & 0.3 & 0.9 \\
4 & estático & 16 & 0.3 & 0.9 \\
5 & estático & 8 & 0.7 & 0.5 \\
6 & estático & 16 & 0.7 & 0.5 \\
7 & estático & 8 & 0.7 & 0.9 \\
8 & estático & 16 & 0.7 & 0.9 \\
9 & estático & Não se aplica \\
10 & random waypoint & 8 & 0.3 & 0.5 \\
11 & random waypoint & 16 & 0.3 & 0.5 \\
12 & random waypoint & 8 & 0.3 & 0.9 \\
13 & random waypoint & 16 & 0.3 & 0.9 \\
14 & random waypoint & 8 & 0.7 & 0.5 \\
15 & random waypoint & 16 & 0.7 & 0.5 \\
16 & random waypoint & 8 & 0.7 & 0.9 \\
17 & random waypoint & 16 & 0.7 & 0.9 \\
18 & random waypoint & Não se aplica \\
\hline
\end{tabular}

Tabela 2. Experimentos realizados.

Equação 5 que ajusta o compromisso entre média de duração dos enlaces e velocidade do dispositivo, e, por fim, o $\beta$ da Equação 6, que faz o balanço do compromisso entre usar ETX e EM. Com base nesses 4 fatores $(k=4)$, foram realizados 16 experimentos, sendo 3 repetições "aleatórias" para os de cenário estático e 2 repetições para os de cenário móvel, diferença essa, devido ao tempo de execução necessários para cada experimento. Foi adicionado um experimento para cada cenário, utilizando somente o ETX, o qual pode ser entendido como uma base de referência inicial de desempenho do MobETX. As configurações de cada experimento estão descritas na Tabela 2. Observe que os cenários representam mobilidade extrema (utilizando o Random Waypoint) e mobilidade nula (nós estáticos). Esses cenários foram escolhidos propositalmente para verificar os limites da abordagem proposta. Desta maneira, espera-se que em cenários de mobilidade moderada o MobETX venha a apresentar resultados satisfatórios.

\subsection{Resultados}

Esta seção apresenta os resultados obtidos a partir dos experimentos fatoriais $2^{k}$. Na Figura4 4 é possível comparar cada cenário experimentado com seu respectivo experimento de referência (aquele que usa somente o ETX), onde seed é uma informação, usada por um software para a criação de um cenário, implicando que a mesma seed, no mesmo programa, em diferentes execuções, irá gerar resultados pseudo aleatórios. Outro meio usado para aleatorizar os resultados foi a utilização de diferentes rastros de mobilidade para cada seed utilizada. Ademais, nas Tabelas 3 e 4 são apresentadas as interações entre os fatores estudados e com isto, como cada um interfere no resultado final do experimento. As colunas $\lambda, \alpha$ e $\beta$, representam as variáveis de configuração das simulações 5 e a coluna $A V G$ representa a média dos resultados obtidos nos experimentos configurados de cordo $\operatorname{com} \lambda, \alpha$ e $\beta$. A última linha das tabelas apresenta o Valor de interação, o qual representa o quanto uma determinada variável ou conjunto delas impacta nos resultados obtidos. Damos destaque para o $\alpha$ e $\beta$ que apresentam os valores mais significativos (realçado nas tabelas), sendo, portanto os fatores que mais afetam os resultados, o que é justo pois, são

\footnotetext{
${ }^{5}$ Variável em +1 para o maior valor e -1 para o menor valor possível para a determinada variável, conforme Tabela 2
} 
exatamente os fatores que ajustam os compromissos referentes à mobilidade das entidades da rede e o balanceamento entre o ETX e a EM.

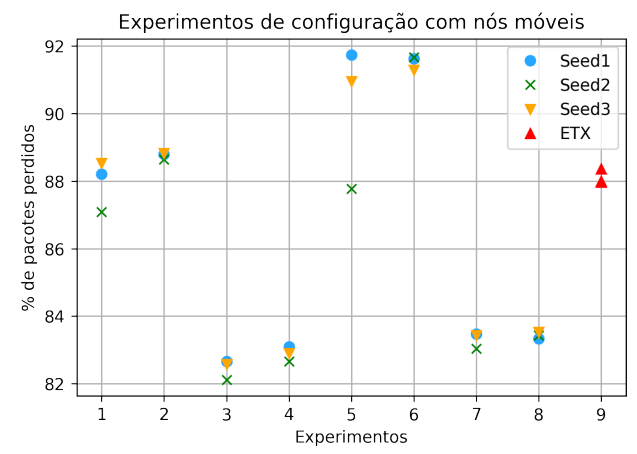

(a)

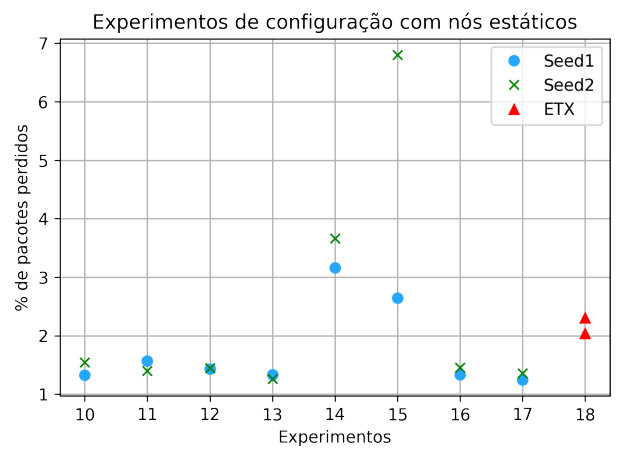

(b)

Figura 4. Experimentos de configuração.

\begin{tabular}{|c|c|c|c|c|c|c|c|c|c|c|}
\hline$\lambda$ & $\alpha$ & $\beta$ & AVG & $\lambda$ & $\alpha$ & $\beta$ & $\lambda \alpha$ & $\lambda \beta$ & $\alpha \beta$ & $\lambda \alpha \beta$ \\
\hline-1 & -1 & -1 & 87,94 & $-87,9$ & $-87,9$ & $-87,9$ & 87,94 & 87,94 & 87,94 & $-87,9$ \\
\hline 1 & -1 & -1 & 88,75 & 8,75 & $-88,8$ & $-88,8$ & 8,8 & $-88,8$ & 88 & 88,75 \\
\hline-1 & -1 & 1 & 82,44 & $-82,4$ & $-82,4$ & 82,44 & 82,44 & $-82,4$ & $-82,4$ & 82,44 \\
\hline 1 & -1 & 1 & 82,88 & 82,88 & $-82,88$ & 82,8 & -82 & 82, & -82 & $-82,88$ \\
\hline-1 & 1 & -1 & 90,1 & -90 & & & & 90,15 & $-90,2$ & 90,15 \\
\hline 1 & 1 & -1 & 91,53 & 91,53 & & -91 & & & & $-91,5$ \\
\hline-1 & 1 & 1 & 83,31 & $-83,3$ & & 83,31 & & $-83,3$ & 83,31 & $-83,3$ \\
\hline 1 & 1 & 1 & 83,42 & 83,42 & 83,42 & 83,42 & & 83,42 & & 83,42 \\
\hline & eração & 0,68 & 1,59 & $-6,57$ & 0,06 & $-0,4$ & $-0,89$ & $-0,22$ \\
\hline
\end{tabular}

Tabela 3. Matriz de efeito dos fatores em cenários móveis.

\begin{tabular}{|c|c|c|c|c|c|c|c|c|c|c|}
\hline$\lambda$ & $\alpha$ & $\beta$ & AVG & $\lambda$ & $\alpha$ & $\beta$ & $\lambda \alpha$ & $\lambda \beta$ & $\alpha \beta$ & $\lambda \alpha \beta$ \\
\hline-1 & -1 & -1 & 1,43 & $-1,43$ & $-1,43$ & $-1,43$ & 1,43 & 1,43 & 1,43 & $-1,43$ \\
\hline 1 & -1 & -1 & 1,48 & 1,48 & $-1,48$ & $-1,48$ & $-1,48$ & $-1,48$ & 1,48 & 1,48 \\
\hline-1 & -1 & 1 & 1,44 & $-1,44$ & $-1,44$ & 1,44 & 1,44 & $-1,44$ & $-1,44$ & 1,44 \\
\hline 1 & -1 & 1 & 1,30 & 1,30 & $-1,30$ & 1,30 & $-1,30$ & 1,30 & $-1,30$ & $-1,30$ \\
\hline-1 & 1 & -1 & 3,41 & $-3,41$ & 3,41 & $-3,41$ & $-3,41$ & 3,41 & $-3,41$ & 3,41 \\
\hline 1 & 1 & -1 & 4,72 & 4,72 & 4,72 & $-4,72$ & 4,72 & $-4,72$ & $-4,72$ & $-4,72$ \\
\hline-1 & 1 & 1 & 1,39 & $-1,39$ & 1,39 & 1,39 & $-1,39$ & $-1,39$ & 1,39 & $-1,39$ \\
\hline 1 & 1 & 1 & 1,30 & 1,30 & 1,30 & 1,30 & 1,30 & 1,30 & 1,30 & 1,30 \\
\hline \multicolumn{4}{|c|}{ Valor de interação } & 0,28 & 1,29 & $-1,40$ & 0,32 & $-0,39$ & $-1,31$ & $-0,30$ \\
\hline
\end{tabular}

Tabela 4. Matriz de efeito dos fatores em cenários estáticos.

Para verificar se a variação dos fatores $\lambda, \alpha$ e $\beta$ são significativos nos resultados obtidos, construímos as Tabelas 5 e 6 ANOVA. A tabela ANOVA tem como objetivo fazer uma análise estatística dos efeitos dos fatores em análise, a partir dos resultados obtidos nos experimentos. Nas Tabelas 5 e 6, a razão F é o resultado obtido na ANOVA a fim de ser comparado com o valor na tabela $\mathrm{F}$ e nos informar se este valor é estatisticamente significativo. Em nossos experimentos, ao se utilizar uma significância de 5\% para ambos os cenários, obtemos na tabela $\mathrm{F}$, o valor de 5,32 no cenário estático e 4,46 para o cenário móvel. Com estes valores, concluímos que a variação dos fatores $\alpha, \beta$ e de sua interação são relevantes no cenário estático e em cenário móvel, a variação de $\alpha \mathrm{e} \beta$ tem efeito significativo em nossos resultados (em destaque nas tabelas). Concluímos, por fim, que $\alpha$ e $\beta$ tem grande relevância no MobETX em ambos os casos, e, portanto, em trabalhos futuros, devem ser considerados na elaboração de novos LQEs de premissa similar.

Após observação das tabelas ANOVA e da Figura 4, o experimento 4, com a configuração que pode ser observada na Tabela 2, foi escolhido como a configuração padrão do MobETX, devido ao seu desempenho em ambas as situações. A partir desta configuração foram realizados 15 experimentos com ambos LQEs a fim de verificar a variância dos resultados e analisar a viabilidade do MobETX. Os resultados destes experimentos podem ser visualizados na Figura 5, onde, no eixo Y, está representado a porcentagem de pacotes perdidos da repetição indicada no eixo $\mathrm{X}$. 


\begin{tabular}{lcccc}
\hline Fator & Soma dos quadrados & graus de liberdade & Quadrado médio & Razão F \\
\hline$\lambda$ & 2,7973677 & 2 & 1,3986838 & 1,0701165 \\
$\alpha$ & 259,6240735 & 2 & 129,8120367 & $\mathbf{9 9 , 3 1 7 6 5 6 5}$ \\
$\beta$ & 15,3482521 & 2 & 7,6741260 & $\mathbf{5 , 8 7 1 3 8 3 2}$ \\
$\lambda \alpha$ & 0,9998594 & 2 & 0,4999297 & 0,3824903 \\
$\lambda \beta$ & 0,0239234 & 2 & 0,0119617 & 0,0091517 \\
$\alpha \beta$ & 4,7842652 & 2 & 2,3921326 & 1,8301924 \\
$\lambda \alpha \beta$ & 0,2991444 & 2 & 0,1495722 & 0,1144359 \\
Erro & 10,4563108 & 8 & 1,3070388 & \\
Total & 294,3331969 & 22 & & \\
\hline
\end{tabular}

Tabela 5. Tabela ANOVA em cenário móvel.

\begin{tabular}{lrrrc}
\hline Fator & Soma dos quadrados & graus de liberdade & Quadrado médio & Razão F \\
\hline$\lambda$ & 0,3195881 & 1 & 0,3195881 & 0,2906174 \\
$\alpha$ & 7,8822810 & 1 & 7,8822810 & $\mathbf{7 , 1 6 7 7 5 0 8}$ \\
$\beta$ & 6,6748314 & 1 & 6,6748314 & $\mathbf{6 , 0 6 9 7 5 6 7}$ \\
$\lambda \alpha$ & 0,6306541 & 1 & 0,6306541 & 0,5734852 \\
$\lambda \beta$ & 0,4278915 & 1 & 0,4278915 & 0,3891030 \\
$\alpha \beta$ & 6,9072746 & 1 & 6,9072746 & $\mathbf{6 , 2 8 1 1 2 8 9}$ \\
$\lambda \alpha \beta$ & 0,3636942 & 1 & 0,3636942 & 0,3307253 \\
Erro & 8,7974944 & 8 & 1,0996868 & \\
Total & 32,0037093 & 15 & & \\
\hline
\end{tabular}

Tabela 6. Tabela ANOVA em cenário estático.

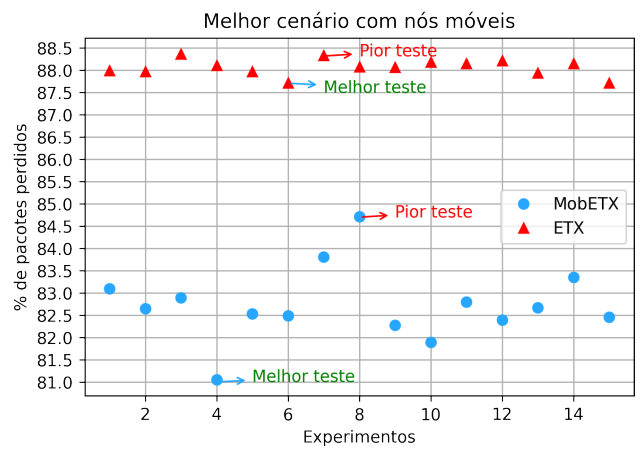

(a)

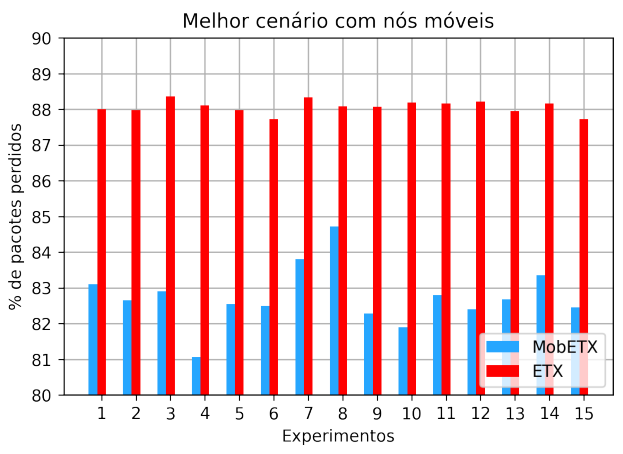

(c)

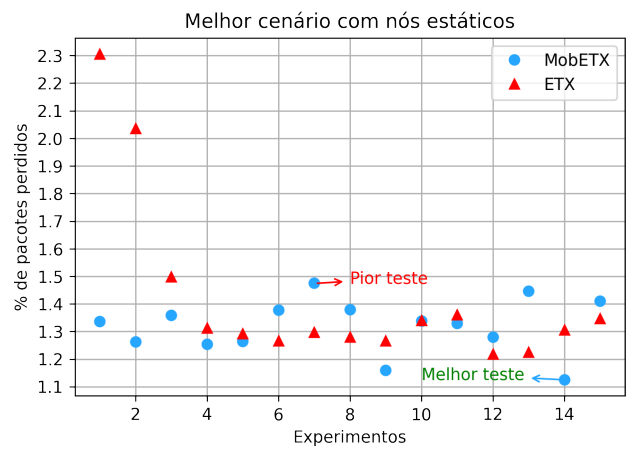

(b)

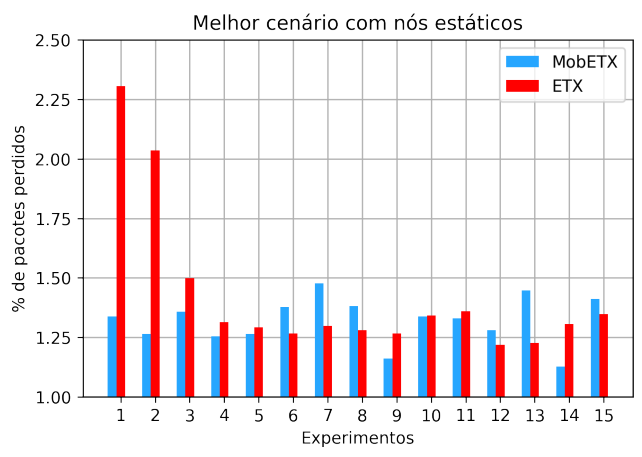

(d)

Figura 5. Experimentos na melhor configuração.

Para finalizar os gráficos de comparação, a Figura 6 apresenta os resultados para se avaliar o intervalo de confiança dos experimentos, com uma confiança de $95 \%$ (distribuição T-Student). Nestes gráficos podemos notar que o MobETX em um cenário móvel apesar de, uma variância maior entre seus resultados (variando 0,91 enquanto o ETX varia 0,20 pontos percentuais), possui uma melhora de aproximadamente $5 \%$ (média de $82,73 \%$ para o MobETX e 88,06\% para o ETX) em relação à porcentagem de perda de pacotes, quando comparado aos resultados obtidos pelo ETX. Em cenário estático, mantém performance similar ao ETX (média de 1,32\% para o MobETX e 1,42\% do ETX). 


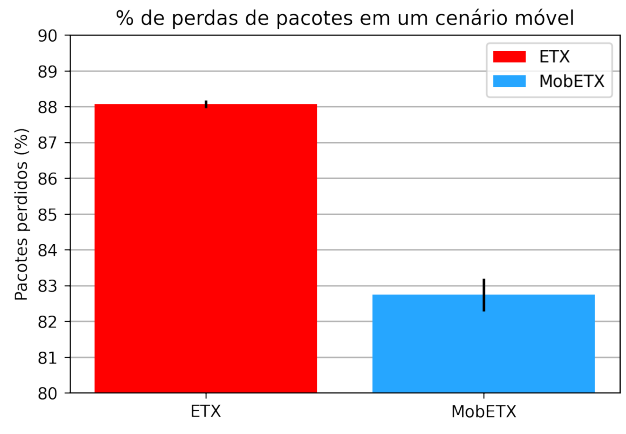

(a)

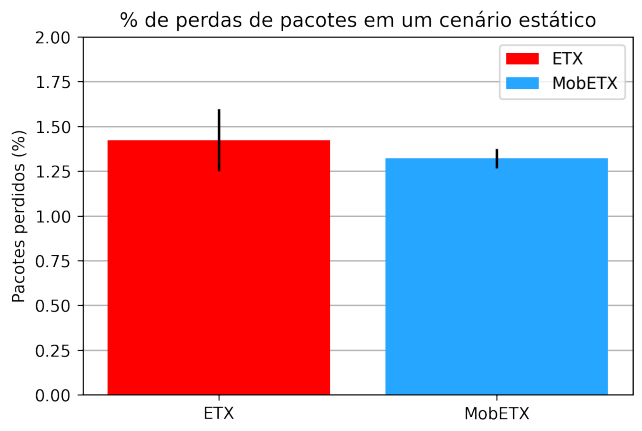

(b)

Figura 6. Porcentagem de pacotes perdidos com a melhor configuração.

\section{Conclusão}

Um novo estimador, chamado MobETX, foi apresentado neste artigo. O MobETX, categorizado como um LQE híbrido, estima a qualidade do enlace utilizando métricas de mobilidade dos nós da rede em conjunto com o ETX para se obter uma melhora na taxa de entrega de pacotes da rede. Foram feitos experimentos, baseados no experimento fatorial $2^{k}$ para definir os valores adequados dos fatores de configuração. Após, foram realizados experimentos a partir da configuração escolhida em cenários com dispositivos móveis e em cenários com dispositivos estáticos, comparando os resultados obtidos com o desempenho do ETX nos mesmos cenários.

Os resultados obtidos indicam que o MobETX superou o ETX em 5\% na taxa de perda de pacotes em cenário com dispositivos móveis, com performance similar em redes com dispositivos estáticos. Estes resultados mostram que o MobETX pode ser uma alternativa promissora ao ETX, visto que além da melhora na perda de pacotes, mantém todas as características do ETX, sem a necessidade de alterações no protocolo de roteamento subjacente como, por exemplo, o RPL.

Futuramente, o MobETX necessitará de experimentos mais complexos de mobilidade como, por exemplo, cenários com múltiplos padrões de mobilidade simultâneos. Ademais, destaca-se validar o MobETX em cenários reais de testbed dado que sua implementação é compatível com o ContikiOS. O MobETX pode ser incorporado com outros estimadores já existentes, a fim de otimizar outros fatores, aqui não abordados, como consumo energético da rede, latência, centralidade, entre outros.

\section{Referências}

Alizai, M. H., Wirtz, H., Kunz, G., Grap, B., and Wehrle, K. (2011). Efficient online estimation of bursty wireless links. In 2011 ISCC. IEEE.

Atzori, L., Iera, A., and Morabito, G. (2010). The internet of things: A survey. Computer networks, 54(15):2787-2805.

Baccour, N., Koubâa, A., Mottola, L., Zúñiga, M. A., Youssef, H., Boano, C. A., and Alves, M. (2012). Radio link quality estimation in wireless sensor networks: A survey. ACM TOSN, 8(4):34. 
Bahr, M., Wang, J., and Jia, X. (2006). Routing in Wireless Mesh Networks. Proceedings of the Annual International Conference on MOBICOM.

Boano, C. A., Zúniga, M. A., Voigt, T., Willig, A., and Römer, K. (2010). The triangle metric: Fast link quality estimation for mobile wireless sensor networks. In ICCCN. IEEE.

Charles, A. J. and Palanisamy, K. (2020). Neo-hybrid Composite Routing Metric for RPL. Procedia Computer Science, 171:1819-1828.

De Couto, D. S., Aguayo, D., Bicket, J., and Morris, R. (2005). A high-throughput path metric for multi-hop wireless routing. Wireless networks.

Ding, W., Tang, L., and Ji, S. (2016). Optimizing routing based on congestion control for wireless sensor networks. Wireless Networks, 22(3):915-925.

Draves, R., Padhye, J., and Zill, B. (2004). Comparison of routing metrics for static multi-hop wireless networks. ACM SIGCOMM CCR.

Dunkels, A., Gronvall, B., and Voigt, T. (2004). Contiki-a lightweight and flexible operating system for tiny networked sensors. In 29th annual IEEE LCN.

Fotouhi, H., Moreira, D., and Alves, M. (2015). mRPL: Boosting mobility in the Internet of Things. Ad Hoc Networks, 26:17-35.

Gnawali, O. and Levis, P. (2012). The minimum rank with hysteresis objective function. RFC 6719.

Guo, Z.-Q., Wang, Q., Li, M.-H., and He, J. (2013). Fuzzy logic based multidimensional link quality estimation for multi-hop wireless sensor networks. IEEE Sensors Journal, 13(10):3605-3615.

Instruments, T. (2007). CC2420 datasheet. Reference SWRS041B.

Jayasri, T. and Hemalatha, M. (2017). Link quality estimation for adaptive data streaming in wsn. Wireless Personal Communications.

Kassab, W. and Darabkh, K. A. (2020). A-Z survey of Internet of Things: Architectures, protocols, applications, recent advances, future directions and recommendations. Journal of Network and Computer Applications, 163:102663.

Lamaazi, H. and Benamar, N. (2017). RPL enhancement using a new objective function based on combined metrics. In 2017 13th IEEE IWCMC.

Liu, D., Cao, Z., Hou, M., and Zhang, Y. (2016). Frame counter: Achieving accurate and real-time link estimation in low power wireless sensor networks. In 2016 15th ACM/IEEE IPSN, pages 1-12. IEEE.

Liu, T. and Cerpa, A. E. (2014a). Data-driven link quality prediction using link features. ACM TOSN, 10(2):1-35.

Liu, T. and Cerpa, A. E. (2014b). Temporal adaptive link quality prediction with online learning. ACM TOSN, 10(3):1-41.

Lowrance, C. J. and Lauf, A. P. (2017). Link quality estimation in ad hoc and mesh networks: a survey and future directions. Wireless Personal Communications. 
Oliveira, A. and Vazao, T. (2016). Low-power and lossy networks under mobility: A survey. Computer networks, 107:339-352.

Pereira, H., Moritz, G. L., and Fonseca, M. (2019). Uma métrica de roteamento com eficiência energética para o $\mathrm{rpl}$ da internet das coisas. In Anais do XXXVII SBRC, pages 224-237. SBC.

Ren, F., He, T., Das, S. K., and Lin, C. (2011). Traffic-aware dynamic routing to alleviate congestion in wireless sensor networks. IEEE TPDS.

Santos, B. P., Goussevskaia, O., Vieira, L. F., Vieira, M. A., and Loureiro, A. A. (2018). Mobile matrix: routing under mobility in IoT, IoMT, and social IoT. Ad Hoc Networks, 78:84-98.

Santos, B. P., Silva, L. A., Celes, C., Borges, J. B., Neto, B. S. P., Vieira, M. A. M., Vieira, L. F. M., Goussevskaia, O. N., and Loureiro, A. (2016). Internet das coisas: da teoria à prática. Minicursos $S B R C$.

Santos, B. P., Vieira, L. F., and Vieira, M. A. (2017). CGR: Centrality-based green routing for Low-power and Lossy Networks. Computer Networks.

Santos, B. P., Vieira, L. F. M., and Loureiro, A. A. F. (2020). Routing and Mobility Management in the Internet of Things. In Anais Estendidos do XXXVIII SBRC, pages 161-168. SBC.

Sobral, J. V., Rodrigues, J. J., Rabêlo, R. A., Al-Muhtadi, J., and Korotaev, V. (2019). Routing Protocols for Low Power and Lossy Networks in Internet of Things Applications. Sensors, 19(9):2144.

Souza, F. R., Domingues, A. C., de Melo, P. O. V., and Loureiro, A. A. (2018). MOCHA: Um framework para caracterização e comparação de traces de mobilidade. In Anais do $X X X V I S B R C$. SBC.

Sun, W., Lu, W., Li, Q., Chen, L., Mu, D., and Yuan, X. (2017). Wnn-lqe: Wavelet-neuralnetwork-based link quality estimation for smart grid wsns. IEEE Access, 5:1278812797.

Tan, W. L., Hu, P., and Portmann, M. (2012). SNR-based link quality estimation. In 2012 IEEE 75th VTC, pages 1-5.

Thubert, P. et al. (2012). Objective function zero for the routing protocol for low-power and lossy networks (rpl).

University of Osnabrück (2016). A Mobility Scenario Generation and Analysis Tool. University of Osnabrück. http://sys.cs.uos.de/bonnmotion/doc/ README.pdf.

Xue, X., Sun, W., Wang, J., Li, Q., Luo, G., and Yu, K. (2020). Rvfl-lqp: Rvfl-based link quality prediction of wireless sensor networks in smart grid. IEEE Access, 8:78297841 . 